\title{
Correction to: Molecular modeling as a design tool for sunscreen candidates: a case study of bemotrizinol
}

\author{
João Victor Teixeira Gomes ${ }^{1}$ - Anne Cherem Peixoto da Silva ${ }^{1} \cdot$ Murilo Lamim Bello ${ }^{1} \cdot$ Carlos Rangel Rodrigues $^{2}$. \\ Bianca Aloise Maneira Corrêa Santos ${ }^{1}$
}

Published online: 6 January 2020

(C) Springer-Verlag GmbH Germany, part of Springer Nature 2020

\section{Correction to: Journal of Molecular Modeling (2019) 25:362}

https://doi.org/10.1007/s00894-019-4237-7

The original version of this article unfortunately contained mistakes. Table 1 was missing and the presentation of Table 2 was incorrect. In Table 2 , the second $[\lambda \exp (\mathrm{nm})]$ and last $\left[\mathrm{MAD}^{\mathrm{a}}\right]$ columns, many values are wrongly in the same cell/line. For example, in column 2, line 2, the first number (342) should be above the other (318). In the version published, these values are together (342318). This error repeats in column 2, lines 3-4 and last column, lines 2-4. The corrected tables are given below.

The original article has been corrected.

The online version of the original article can be found at https://doi.org/ 10.1007/s00894-019-4237-7

Bianca Aloise Maneira Corrêa Santos

bialoise@pharma.ufrj.br

1 Laboratório de Planejamento Farmacêutico e Simulação Computacional (LaPFarSC), Faculdade de Farmácia, Universidade Federal do Rio de Janeiro, Rio de Janeiro, RJ 21941-902, Brazil

2 Laboratório de Modelagem Molecular \& QSAR (ModMolQSAR), Faculdade de Farmácia, Universidade Federal do Rio de Janeiro, Rio de Janeiro, RJ 21941-902, Brazil 
Table 1 Total energy (eV) and structural parameters ${ }^{\mathrm{a}}$ of the three lower energy geometries (conf 1-3) of each triazine derivatives (A-D) obtained by ground-stated optimization at DFT/B3LYP/6-31G(d)

\begin{tabular}{|c|c|c|c|c|}
\hline \multirow{2}{*}{ Derivatives } & \multirow{2}{*}{ Data } & \multicolumn{3}{|c|}{ Conformers } \\
\hline & & conf1 & $\operatorname{conf} 2$ & $\operatorname{conf} 3$ \\
\hline \multirow{4}{*}{$\mathbf{A}$} & $\mathrm{eV}$ & -49748.65 & -49748.65 & -49748.67 \\
\hline & $\alpha_{1}$ & 0.74 & -0.46 & 0.85 \\
\hline & $\alpha_{2}$ & -0.44 & -0.91 & -0.97 \\
\hline & $\alpha_{3}$ & -1.28 & -1.09 & -0.75 \\
\hline \multirow{5}{*}{ B } & $\mathrm{eV}$ & -52865.47 & -52865.34 & -52865.32 \\
\hline & $\mathrm{OH}^{1 \cdots N} \mathrm{~N}$ & 1.708 & 1.705 & 1.715 \\
\hline & $\alpha_{1}$ & -2.56 & -2.6 & -3.63 \\
\hline & $\alpha_{2}$ & -9.19 & -10.65 & -11.03 \\
\hline & $\alpha_{3}$ & -1.86 & -0.56 & -2.53 \\
\hline \multirow{7}{*}{$\mathbf{C}$} & $\mathrm{eV}$ & -56959.44 & -56959.41 & -56959.41 \\
\hline & $\mathrm{OH}^{1 \cdots} \mathrm{N}$ & 1.711 & 1.708 & 1.705 \\
\hline & $\mathrm{OH}^{2 \cdots} \mathrm{N}$ & 1.707 & 1.705 & 1.711 \\
\hline & $\mathrm{OH}^{3 \cdots} \mathrm{N}$ & 1.703 & 1.722 & 1.720 \\
\hline & $\alpha_{1}$ & -7.27 & 7.06 & 6.8 \\
\hline & $\alpha_{2}$ & -7.76 & 7.57 & 7.95 \\
\hline & $\alpha_{3}$ & -6.86 & 8.38 & 8.03 \\
\hline \multirow{6}{*}{ D (BEMT) } & $\mathrm{eV}$ & -54912.45 & -54912.36 & -54912.41 \\
\hline & $\mathrm{OH}^{2 \cdots} \mathrm{N}$ & 1.710 & 1.709 & 1.712 \\
\hline & $\mathrm{OH}^{3 \cdots} \mathrm{N}$ & 1.704 & 1.704 & 1.700 \\
\hline & $\alpha_{1}$ & -10.73 & 18.46 & 10.34 \\
\hline & $\alpha_{2}$ & -2.84 & 3.33 & 2.65 \\
\hline & $\alpha_{3}$ & -7.19 & 1.9 & 6.87 \\
\hline
\end{tabular}

${ }^{\mathrm{a}}$ The $\mathrm{H}$ bond lengths are in $\AA$ and dihedral angles $\alpha$ between the benzene and triazine rings in ${ }^{\circ}$ (degrees). 
Table 2 Transitions $(\mathrm{eV} / \mathrm{nm})$ and oscillator strength $(f)$ calculated for the ten lowest states in gas phase, the experimental absorption maxima ( $\lambda$ exp) in ethanol [17] and the mean absolute deviation (MAD) ${ }^{\mathrm{a}}$ between the experimental $(\lambda \exp )$ and calculated $(\lambda \text { calc })^{\mathrm{b}}$ absorptions maxima for the UVA and UVB ranges of derivatives A-D

\begin{tabular}{|c|c|c|c|c|c|c|c|c|c|c|c|}
\hline \multirow[t]{2}{*}{ Derivative } & \multirow[t]{2}{*}{$\lambda \exp (n m)$} & \multicolumn{3}{|l|}{ conf1 } & \multicolumn{3}{|l|}{$\operatorname{conf} 2$} & \multicolumn{3}{|l|}{ conf3 } & \multirow[t]{2}{*}{$\mathrm{MAD}^{\mathrm{a}}$} \\
\hline & & $\mathrm{eV}$ & $\mathrm{nm}$ & $f$ & $\mathrm{eV}$ & $\mathrm{nm}$ & $f$ & $\mathrm{eV}$ & $\mathrm{nm}$ & $f$ & \\
\hline \multirow[t]{10}{*}{ A } & \multirow[t]{10}{*}{310} & 3.775 & 328 & 0.000 & 3.775 & 328 & 0.000 & 3.775 & 328 & 0.000 & \multirow[t]{10}{*}{2.00} \\
\hline & & 3.964 & 313 & 0.003 & 3.957 & 313 & 0.008 & 3.962 & 313 & 0.017 & \\
\hline & & 3.981 & 311 & 0.041 & 3.986 & 311 & 0.020 & 3.983 & 311 & 0.003 & \\
\hline & & 4.017 & 309 & 1.062 & 4.017 & 309 & 0.924 & 4.016 & 309 & 1.010 & \\
\hline & & 4.044 & 307 & 0.816 & 4.046 & 306 & 0.966 & 4.044 & 307 & 0.881 & \\
\hline & & 4.151 & 299 & 0.001 & 4.151 & 299 & 0.001 & 4.150 & 299 & 0.003 & \\
\hline & & 4.216 & 294 & 0.000 & 4.212 & 294 & 0.001 & 4.214 & 294 & 0.001 & \\
\hline & & 4.219 & 294 & 0.000 & 4.218 & 294 & 0.000 & 4.217 & 294 & 0.000 & \\
\hline & & 4.227 & 293 & 0.005 & 4.233 & 293 & 0.004 & 4.228 & 293 & 0.004 & \\
\hline & & 4.302 & 288 & 0.025 & 4.298 & 288 & 0.010 & 4.300 & 288 & 0.017 & \\
\hline \multirow[t]{10}{*}{ B } & \multirow{10}{*}{$\begin{array}{l}342 \\
318\end{array}$} & 3.400 & 365 & 0.002 & 3.404 & 364 & 0.002 & 3.492 & 355 & 0.003 & \multirow{10}{*}{$\begin{array}{l}5.00 \\
12.67\end{array}$} \\
\hline & & 3.662 & 339 & 0.481 & 3.680 & 337 & 0.465 & 3.700 & 335 & 0.347 & \\
\hline & & 3.837 & 323 & 0.180 & 3.830 & 324 & 0.212 & 3.817 & 325 & 0.107 & \\
\hline & & 3.981 & 311 & 0.299 & 3.977 & 312 & 0.161 & 3.894 & 318 & 0.106 & \\
\hline & & 4.007 & 309 & 0.465 & 4.000 & 310 & 0.698 & 3.966 & 313 & 0.358 & \\
\hline & & 4.087 & 303 & 0.017 & 4.093 & 303 & 0.033 & 3.984 & 311 & 0.410 & \\
\hline & & 4.131 & 300 & 0.055 & 4.120 & 301 & 0.006 & 4.042 & 307 & 0.630 & \\
\hline & & 4.146 & 299 & 0.473 & 4.149 & 299 & 0.374 & 4.132 & 300 & 0.003 & \\
\hline & & 4.280 & 290 & 0.005 & 4.294 & 289 & 0.006 & 4.258 & 291 & 0.012 & \\
\hline & & 4.295 & 289 & 0.003 & 4.309 & 288 & 0.027 & 4.279 & 290 & 0.004 & \\
\hline \multirow[t]{10}{*}{$\mathrm{C}$} & \multirow{10}{*}{$\begin{array}{l}362 \\
305\end{array}$} & 3.426 & 362 & 0.002 & 3.433 & 361 & 0.006 & 3.430 & 361 & 0.007 & \multirow{10}{*}{$\begin{array}{l}17.33 \\
4.00\end{array}$} \\
\hline & & 3.474 & 357 & 0.124 & 3.478 & 356 & 0.109 & 3.480 & 356 & 0.113 & \\
\hline & & 3.485 & 356 & 0.215 & 3.521 & 352 & 0.319 & 3.519 & 352 & 0.352 & \\
\hline & & 3.580 & 346 & 0.442 & 3.592 & 345 & 0.418 & 3.600 & 344 & 0.417 & \\
\hline & & 3.590 & 345 & 0.463 & 3.608 & 344 & 0.281 & 3.602 & 344 & 0.245 & \\
\hline & & 3.766 & 329 & 0.001 & 3.733 & 332 & 0.037 & 3.733 & 332 & 0.038 & \\
\hline & & 4.084 & 304 & 0.041 & 3.894 & 318 & 0.008 & 3.895 & 318 & 0.004 & \\
\hline & & 4.090 & 303 & 0.378 & 3.971 & 312 & 0.471 & 3.969 & 312 & 0.501 & \\
\hline & & 4.108 & 302 & 0.356 & 4.094 & 303 & 0.189 & 4.088 & 303 & 0.004 & \\
\hline & & 4.124 & 301 & 0.055 & 4.101 & 302 & 0.220 & 4.095 & 303 & 0.367 & \\
\hline \multirow[t]{10}{*}{ D (BEMT) } & \multirow{10}{*}{$\begin{array}{l}343 \\
310\end{array}$} & 3.167 & 391 & 0.152 & 3.373 & 368 & 0.012 & 3.384 & 366 & 0.030 & \multirow{10}{*}{$\begin{array}{l}9.67 \\
3.00\end{array}$} \\
\hline & & 3.405 & 364 & 0.033 & 3.387 & 366 & 0.001 & 3.467 & 358 & 0.019 & \\
\hline & & 3.416 & 363 & 0.391 & 3.558 & 348 & 0.811 & 3.574 & 347 & 0.615 & \\
\hline & & 3.536 & 351 & 0.056 & 3.719 & 333 & 0.146 & 3.724 & 333 & 0.337 & \\
\hline & & 3.725 & 333 & 0.339 & 3.986 & 311 & 0.082 & 3.964 & 313 & 0.068 & \\
\hline & & 3.812 & 325 & 0.007 & 4.014 & 309 & 0.092 & 4.028 & 308 & 0.313 & \\
\hline & & 3.977 & 312 & 0.496 & 4.085 & 304 & 0.228 & 4.069 & 305 & 0.237 & \\
\hline & & 4.024 & 308 & 0.000 & 4.118 & 301 & 0.567 & 4.109 & 302 & 0.138 & \\
\hline & & 4.099 & 302 & 0.193 & 4.138 & 300 & 0.099 & 4.159 & 298 & 0.250 & \\
\hline & & 4.340 & 286 & 0.025 & 4.298 & 288 & 0.004 & 4.243 & 292 & 0.010 & \\
\hline
\end{tabular}

${ }^{\mathrm{a}} \sum|\lambda \exp -\lambda \mathrm{calc}|$

${ }^{\mathrm{b}}$ The calculated absorption maxima ( $\lambda$ calc) at UVA and UVB ranges is in bold 